\title{
Gender Identification from Facial Image using Compound Local Binary Pattern (CLBP)
}

\author{
Emam Hossain \\ Ahsanullah University of \\ Science and Technology, \\ Dhaka, Bangladesh
}

\author{
Shayla Azad Bhuyan \\ BRAC University, Dhaka, \\ Bangladesh
}

\author{
Faisal Ahmed \\ Islamic University of \\ Technology, Dhaka, \\ Bangladesh
}

\begin{abstract}
Automatic identification of male and female from facial image allows many useful applications in biometrics, surveillance, and human-computer interaction. This paper presents a robust face feature descriptor for gender classification from facial image. The proposed method is based on the compound local binary pattern (CLBP), an extension of the LBP texture operator. The CLBP operator exploits $2 P$ bits to encode the information of a local neighborhood of $P$ neighbors, where $P$ bits are used to express the sign information and the other $P$ bits are used to express the magnitude information of the differences between the center and the neighbor gray values. The performance of the proposed method has been evaluated using a large dataset comprising 1800 facial images collected from the FERET database. Extensive experiments with support vector machine classifier show the superiority of the CLBP feature descriptor against some well-known texture operators.
\end{abstract}

\section{General Terms}

Computer Vision, Pattern Recognition.

\section{Keywords}

Compound LBP (CLBP), Local texture, Gender classification, Support vector machine (SVM).

\section{INTRODUCTION}

With the increasing demand for personalized interaction with consumer products and applications [1], automated gender classification has attracted much attention over the last ten years since augmenting this ability with applications specific to a particular gender can provide a more user-friendly environment and human-like interaction [2], [3]. Since visual information from human face has an important role in gender perception [4], classification of male and female from facial images is being widely studied and has potential applicability in biometrics, surveillance, social robotics, and humancomputer interaction systems. In addition, automated gender classification can also be applied in face recognition applications as a pre-processing step, which can significantly reduce the number of candidates to be matched [5].

A system that classifies gender from facial images generally comprises two fundamental components: a facial feature extractor and a classifier. Designing an effective feature extractor that generates the facial feature vector for classifier training and testing is critical for any successful gender classification system. Although much work has been done, the inherent variability of facial images caused by different factors like variations in illumination, pose, alignment, occlusion, facial expression, and aging makes robust feature extraction a difficult and challenging task [6]. Hence, extraction of facial feature is an active research topic in computer vision, where the aim is to make the features robust against these factors [7]. A few surveys on facial feature representations for face recognition [8] and expression analysis [9] addressed these challenges and possible solutions in detail. Later, Makinen and Raisamo [10], [11] presented experimental evaluation of different gender classification methods.

Based on the types of features used, facial feature extraction approaches can be roughly divided into two different categories: geometric feature-based methods and appearancebased methods [2], [12]. In geometric feature-based methods, the feature vector is formed based on the geometric relationships, such as positions, angles or distances between different facial components [1]. Brunelli and Poggio [13] investigated a set of 16 geometric features for designing a gender classification model. The features were extracted with HyperBF networks. Later, Abdi et al. [14] experimented with a radial basis function (RBF) network and a perceptron, and achieved good classification result for both pixel-based inputs and measurement-based inputs (geometric features). However, the effectiveness of geometric methods is heavily dependent on the accurate detection of facial components, which is a difficult task in changing environment, thus making geometric methods difficult to accommodate in many scenarios [15].

Appearance-based methods extract the facial appearance by applying image filter or filter bank on the whole face image or some specific facial regions. Some widely-used appearancebased methods for automated gender classification include principal component analysis (PCA) [16], independent component analysis (ICA) [17], and Gabor wavelets [18]. Although PCA and ICA feature descriptors can effectively capture the variability of the training images, their performances deteriorate in changing environment [19-21]. On the other hand, convoluting facial images with multiple Gabor filters of many scales and orientations to extract Gabor features is computationally expensive [22]. Recently, local appearance face descriptors based on local binary pattern (LBP) [23], [24] and its variants [12], [25] have attained much attention due to their superior performances in uncontrolled environment. Local binary pattern is a simple, yet effective local texture description technique, which is computationally efficient and robust against monotonic illumination variation. Later, local ternary pattern (LTP) [6] was introduced to increase the robustness of LBP in uniform and near-uniform regions by adding an extra intensity discrimination level and extending the binary LBP value to a ternary code. More recently, Sobel-LBP [19] has been proposed to improve the performance of LBP by applying Sobel operator to enhance the edge information prior to applying LBP for feature extraction. However, in uniform and near-uniform regions, 
Sobel-LBP generates inconsistent patterns as it uses only two discrimination levels just like LBP. Another approach using derivative-based local texture pattern [5] takes the advantage of detailed high-order derivative descriptions and keeps the spatial relationships in local regions. Local directional pattern (LDP), introduced by Jabid et al. [1], [15], [26] employs a different texture encoding approach, where directional edge response values around a position is used instead of gray levels. Although this approach achieves better recognition performance than local binary pattern, LDP tends to produce inconsistent codes in uniform and near-uniform facial regions and is heavily dependent on the selection of the number of prominent edge directions [21], [27].

In our previous work [12], we investigated the representation of face images by means of a new local texture pattern, the compound local binary pattern (CLBP), which yielded better performance for facial expression recognition than some wellknown local texture descriptors. Unlike the original LBP operator that uses $P$ bits to encode only the signs of the differences between the center pixel and $P$ neighbor gray values, the proposed method employs $2 P$ bits, where the additional $P$ bits are used to encode the magnitude information of the differences between the center and the neighbor gray values in a local neighborhood using a threshold. The motivation behind the proposed encoding scheme is to increase the robustness of the texture feature representation by incorporating additional information that is discarded by the original LBP operator. In this paper, we empirically evaluate the performance of the CLBP face descriptor for gender recognition from facial image. Experiments with 1800 facial images collected from the FERET face image database [28] validate the superiority of our proposed method for automated gender identification.

\section{LOCAL BINARY PATTERN (LBP): A REVIEW}

In this section, the local binary pattern (LBP) and the local ternary pattern (LTP) feature extractors are described in details. Local binary pattern (LBP) is a simple, yet effective local texture description technique. LBP was originally introduced by Ojala et al. [29] for grayscale and rotationinvariant texture analysis. Later, many researchers have successfully adopted LBP in different face-related problems, such as face recognition [30] and facial expression analysis [31]. The basic LBP method operates on a local neighborhood around each pixel of an image and thresholds the neighbor gray levels with respect to the center. The result is then concatenated binomially and the center pixel is labeled with the resultant value. Formally, the LBP operator can be represented as:

$$
\begin{aligned}
& \operatorname{LBP}_{P, R}\left(x_{c}, y_{c}\right)=\sum_{p=0}^{P-1} s\left(i_{p}-i_{c}\right) 2^{p} \\
& s(x)= \begin{cases}1, & x \geq 0 \\
0, & x<0\end{cases}
\end{aligned}
$$

Here, $i_{c}$ is the gray value of the center pixel $\left(x_{c}, y_{c}\right), i_{p}$ is the gray value of its neighbors, $P$ is the number of neighbors and $R$ is the radius of the neighborhood. In practice, the LBP operator considers the signs of the differences of the gray values of $P$ equally spaced neighbors with respect to the central pixel in a local neighborhood, which is then represented using a $P$-bit binary number [12]. Bilinear interpolation is used to estimate the gray level of a neighbor if it does not fall exactly on a pixel position [29]. The LBP histogram of the encoded image or image block is then used as the texture feature descriptor. The basic LBP encoding

\begin{tabular}{|c|c|c|c|c|c|c|}
\hline 90 & 32 & 27 & \multirow{3}{*}{ Threshold } & 1 & $\underline{0}$ & $\mathbf{4}^{0}$ \\
\hline 58 & 50 & 12 & & 1 & $C$ & 10 \\
\hline 61 & 38 & 46 & & $1^{\prime}$ & $\overline{0}$ & 0 \\
\hline
\end{tabular}
process is illustrated in Fig 1.

(a)

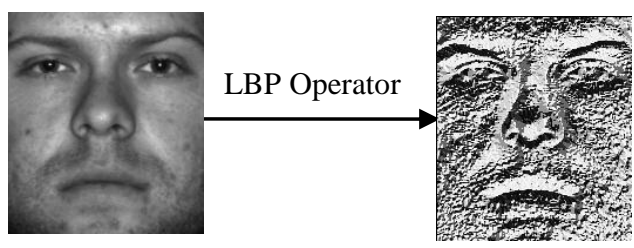

(b)

Fig 1: (a) Illustration of the basic $\mathrm{LBP}_{8,1}$ encoding scheme. Here, the $\mathrm{LBP}_{8,1}$ code 01110000 is used to label the center pixel C, (b) Sample LBP encoded image.

\section{COMPOUND LBP (CLBP)}

The original LBP operator discards the magnitude information of the difference between the center and the neighbor gray values in a local neighborhood. As a result, this method tends to produce inconsistent codes. One example is shown in Fig 2. Here, the 8-bit uniform LBP code (11111111) corresponds to a flat area or a dark spot at the center pixel [16], which is not

\begin{tabular}{|c|c|c|c|c|c|c|}
\hline 50 & 51 & 101 & \multirow{2}{*}{ Threshold } & 1 & 1 & 1 \\
\hline 50 & 50 & 100 & & 11 & $C$ & $\begin{array}{llll}i & 1\end{array}$ \\
\hline 51 & 52 & 102 & & 1 & $-\overline{1}$ & 1 \\
\hline
\end{tabular}
correct in this case.

Fig 2: Generation of inconsistent binary pattern in LBP encoding process.

As LBP operator considers only the sign of the difference between two gray values, it often fails to generate appropriate binary code. Being motivated by this, we propose CLBP, an extension of the original LBP operator that assigns a $2 P$-bit code to the center pixel based on the gray values of a local neighborhood comprising $P$ neighbors. Unlike the LBP that employs one bit for each neighbor to express only the sign of the difference between the center and the corresponding neighbor gray values, the proposed method uses two bits for each neighbor in order to represent the sign as well as the magnitude information of the difference between the center and the neighbor gray values. Here, the first bit represents the sign of the difference between the center and the corresponding neighbor gray values like the basic LBP pattern and the other bit is used to encode the magnitude of the difference with respect to a threshold value, the average magnitude $\left(M_{\text {avg }}\right)$ of the difference between the center and the neighbor gray values in the local neighborhood of interest. The CLBP operator sets this bit to 1 if the magnitude of the difference between the center and the corresponding neighbor is greater than the threshold $M_{\text {avg. }}$. Otherwise, it is set to 0 . Thus, the indicator $s(x)$ of (2) is replaced by the following function: 
$s\left(i_{p}, i_{c}\right)=\left\{\begin{array}{lll}00 & i_{p}-i_{c}<0, & \left|i_{p}-i_{c}\right| \leq M_{\text {avg }} \\ 01 & i_{p}-i_{c}<0, & \left|i_{p}-i_{c}\right|>M_{\text {avg }} \\ 10 & i_{p}-i_{c} \geq 0, & \left|i_{p}-i_{c}\right| \leq M_{\text {avg }} \\ 11 & \text { otherwise }\end{array}\right.$

Here, $i_{c}$ is the gray value of the center pixel, $i_{p}$ is the gray value of a neighbor $p$, and $M_{\text {avg }}$ is the average magnitude of the difference between $i_{p}$ and $i_{c}$ in the local neighborhood. The CLBP operator is illustrated in Fig 3. It can be observed that, the proposed method discriminates the neighbors in the northeast, east, and south-east directions as they have higher gray values than the other neighbors and thus produces a consistent local pattern.

\begin{tabular}{|c|c|c|c|c|c|c|}
\hline 50 & 51 & 101 & \multirow{2}{*}{ Threshold } & 10 & -10 & $\mathbf{4}^{11}$ \\
\hline 50 & 50 & 100 & & $10 !$ & $C$ & 111 \\
\hline 51 & 52 & 102 & & 10 & $\overline{10}$ & 11 \\
\hline
\end{tabular}

\section{Fig 3: Illustration of the basic CLBP operator. Here, the} generated CLBP code is $\mathbf{1 0 1 1 1 1 1 1 1 0 1 0 1 0 1 0}$

In a $3 \times 3$ neighborhood, the proposed CLBP method encodes an image by operating on the 8 neighbors around the central pixel and assigning a 16-bit code to that pixel. As 16-bit codes are used to label the pixels, the number of possible binary patterns is $2^{16}$. To reduce the number of features, $\mathrm{He}$ and Cercone [32] have proposed to consider less number of neighbors while forming the binary patterns. Thus, this method discards some neighborhood information in order to reduce the length of the feature vector. Here, we have proposed a different approach where all the CLBP binary patterns are further split into two sub-CLBP patterns. Each sub-CLBP pattern is obtained by concatenating the bit values corresponding to $P / 2$ neighbors, where $P$ is the number of neighbors. Formally, in a local neighborhood, the two subCLBP patterns are formed by concatenating the corresponding values of the bit sequence $(1,2,5,6, \ldots, 2 P-3,2 P-2)$ and $(3$, $4,7,8, \ldots, 2 P-1,2 P)$, respectively of the $2 P$-bit original CLBP code.

In other words, a 16-bit CLBP pattern is split into two 8-bit sub-CLBP patterns, where the first one is obtained by concatenating the bit values corresponding to the neighbors in the north, east, south, and west directions, respectively and the second sub-CLBP pattern is obtained by concatenating the bit values corresponding to the neighbors in the north-east, southeast, south-west, and north-west directions, respectively. Thus, this method reduces the number of possible patterns significantly, which results in a total of $2^{8}$ distinct sub-CLBP patterns. The process is illustrated in Figure 4. The two subCLBP patterns are treated as separate binary codes and combined during the feature vector generation.

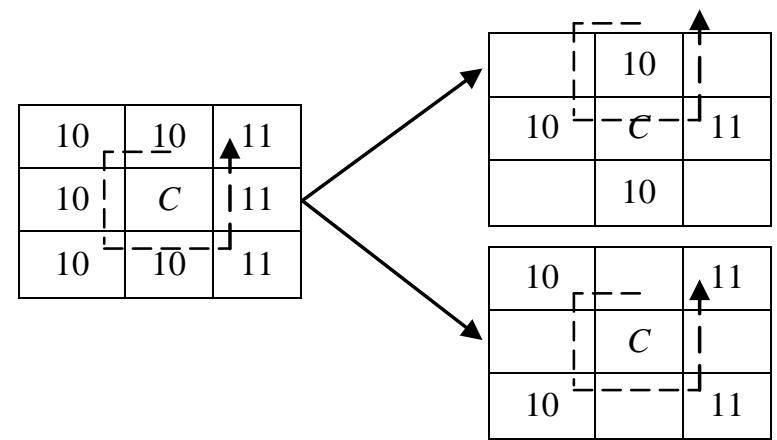

Fig 4: Generation of the sub-CLBP patterns from the original CLBP code

\section{CLBP FEATURE DESCRIPTOR}

After applying the CLBP operator on all the pixels of an image and splitting all the 16-bit CLBP patterns into the corresponding sub-CLBP patterns, we get two 8-bit binary codes for each pixel. Thus, two encoded image representations are obtained for the two sub-CLBP patterns. Two separate histograms are then calculated on the two encoded images, where each of the histograms comprises $2^{8}$ bins. In order to obtain the feature vector, the two histograms are concatenated to obtain a spatially combined histogram. This combined histogram is used as the feature vector that represents the texture information of the image. Each feature vector contains $2 \times 2^{8}$ features. The overall process is shown in Fig 5.

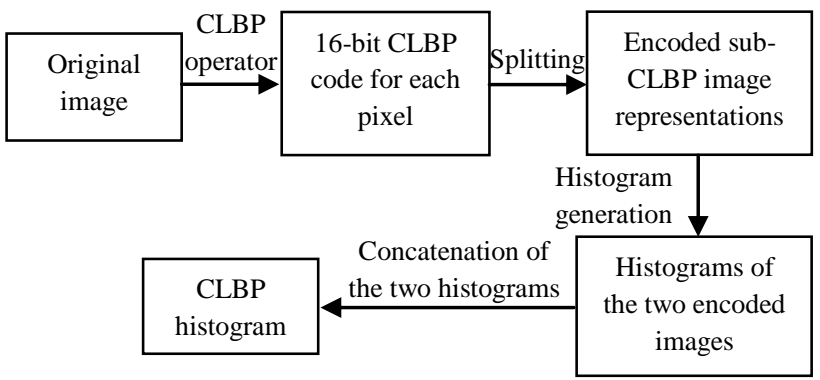

Fig 5: Proposed feature vector generation process using the CLBP operator

Histograms generated from the whole encoded image contain no location information of the micro-patterns, but merely their occurrences are expressed [13], [27], [33]. Therefore, the CLBP histogram is modified to an extended histogram in order to incorporate some degree of location information. First, each image is partitioned into a number of regions and individual CLBP histograms are generated from each of those regions. Finally, the histograms of all the regions are concatenated to obtain the extended CLBP histogram. For the facial expression recognition process, this histogram collection is used as the facial feature vector.

\section{EXPERIMENTAL RESULTS}

The effectiveness of the proposed method for gender classification was evaluated with the face images from the FERET face database [28]. The database is comprised of a total of 14,051 face images representing 1,199 individuals. All the images are frontal, with variations in illumination, pose, facial expression, and aging effect. For our experiment, we selected a total of 1800 images, among which 900 were male and the rest 900 were female face images. The selected images were cropped from the original $512 \times 768$ pixel images 
using the ground truth of the two eye positions and then normalized to $100 \times 100$ pixels. We carried out a ten-fold cross-validation to measure the classification rate. In a tenfold cross-validation, the whole dataset is randomly partitioned into ten subsets, where each subset comprises an equal number of instances. One subset is used as the testing set and the classifier is trained on the remaining nine subsets. The average classification rate is calculated after repeating the above process for ten times.
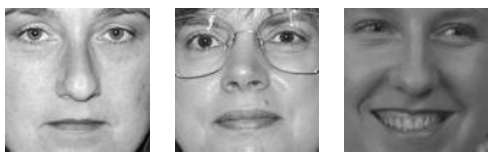

(a)
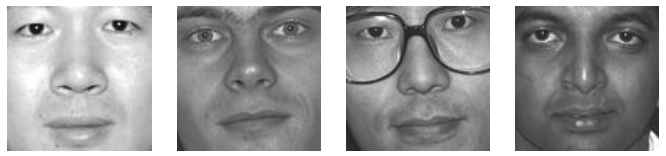

(b)

Fig 6: Sample images from the FERET face database, (a) Female face, (b) Male face.

We have compared the proposed method in terms of classification rate with some widely-used local texture operators, namely local binary pattern (LBP) [23], local directional pattern [26], and local ternary pattern (LTP) [6]. Support vector machine was used for the classification task. Experiments were carried out on images partitioned into $7 \times 7$ sub-regions. Results obtained from the experiments are shown in Table 1. Fig 7 shows the feature vector length of the selected feature descriptors.

Table 1. Classification rate of different feature representation methods using support vector machine (SVM)

\begin{tabular}{|c|c|c|c|}
\hline \multirow{2}{*}{ Method } & \multicolumn{3}{|c|}{ Classification rate (\%) } \\
\cline { 2 - 4 } & Male & Female & Overall \\
\hline $\mathrm{LBP}_{8,1}$ & 92.78 & 92.78 & 92.78 \\
\hline $\mathrm{LTP}$ & 92.89 & 92.89 & 92.89 \\
\hline $\mathrm{LDP}$ & 89.81 & 90.08 & 89.94 \\
\hline $\mathrm{CLBP}_{8,1}$ & 93.87 & 94.07 & 93.97 \\
\hline
\end{tabular}

From the experimental results, it is evident that, facial feature representation based on compound local binary pattern is more robust and this method provides higher classification rate than some existing methods for feature representation. The superiority of the CLBP encoding is due to the utilization of the magnitude of the difference between the center and the neighbor gray values by combining it with the basic LBP pattern, which acts as a compensation for the texture information discarded by the LBP operator. Thus, this method provides an effective and efficient approach to gender classification that is more discriminative than the original LBP operator and outperforms several existing texture description methods.

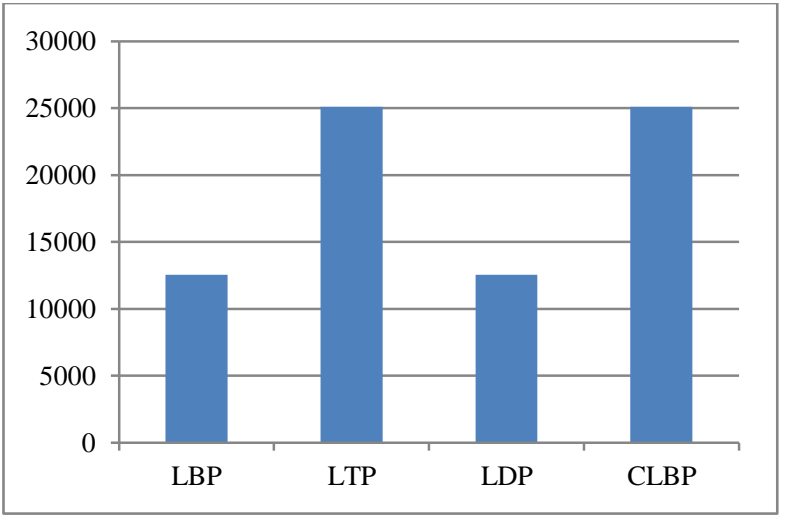

Fig 7: Feature vector length for the different face descriptors

\section{CONCLUSION}

This paper describes the CLBP, an extension of the original LBP operator and a feature representation method based on CLBP codes for gender classification. The proposed method utilizes an encoding scheme that combines the magnitude information of the difference between two gray values with the original LBP pattern and thus provides increased robustness in many situations where LBP fails to generate consistent codes. Experimental results show that, the CLBP operator provides an effective and efficient approach for representing texture information with high discriminative ability.

\section{REFERENCES}

[1] T. Jabid, M. H. Kabir, O. Chae, "Local directional pattern (ldp) for face recognition," International Journal of Innovative Computing, Information and Control, Vol. 8, No. 4, pp. 2423-2437, 2012.

[2] H. C. Kim, D. Kim, Z. Ghahramani, S. Y. Bang, "Appearance-based gender classification with gaussian processes," Pattern Recognition Letters, Vol. 27, No. 6, pp. 618-626, 2006.

[3] F. Ahmed, M. H. Kabir, "Facial feature representation with directional ternary pattern (dtp): Application to gender classification," in Proceedings of the IEEE International Conference on Information Reuse and Integration, USA, 2012, pp. 159-164.

[4] A. B. A. Graf, F. A. Wichmann, "Gender classification of human faces," in Proceedings of the International Workshop on Biologically Motivated Computer Vision, LNCS 2525, 2002, pp. 1-8.

[5] A. Shobeirinejad, Y. Gao, "Gender classification using interlaced derivative patterns," in Proceedings of the International Conference on Pattern Recognition, 2010, pp. 1509-1512.

[6] X. Tan, B. Triggs, "Enhanced local texture feature sets for face recognition under difficult lighting conditions," in Proceedings of the IEEE International Workshop on Analysis and Modeling of Faces and Gestures, LNCS 4778, 2007, pp. 168-182.

[7] F. Ahmed, "Gradient directional pattern: a robust feature descriptor for facial expression recognition," IET Electronics Letters, Vol. 48, No. 19, 2012, pp. 1203 1204. 
[8] W. Zhao, R. Chellappa, P. J. Phillips, "Face recognition: A literature survey," ACM Computing Survey, Vol. 35, No. 4, pp. 399-458, 2003.

[9] B. Fasel, J. Luettin, "Automatic facial expression analysis: A survey," Pattern Recognition, Vol. 36, No. 1, pp. 259-275, 2003.

[10] E. Makinen, R. Raisamo, "An experimental comparison of gender classification methods," Pattern Recognition Letters, Vol. 29, pp. 1544-1556, 2008.

[11] E. Makinen, "Evaluation of gender classification methods with automatically detected and aligned faces," IEEE Transactions on Pattern Analysis and Machine Intelligence, Vol. 30, pp. 541-547, 2008.

[12] F. Ahmed, H. Bari, E. Hossain, "Person-independent facial expression recognition based on compound local binary pattern (clbp)," International Arab Journal of Information Technology, Vol. 11, No. 2, 2013.

[13] R. Brunelli, T. Poggio, "Hyperbf networks for gender classification," in Proceedings of the DARPA Image Understanding Workshop, 1992, pp. 311-314.

[14] H. Abdi, D. Valentin, B. Edelman, A. O'Toole, "More about the difference between men and women: Evidence from linear neural network and principal component approach," Neural Computing, Vol. 7, No. 6, pp. 11601164,1995

[15] T. Jabid, M. H. Kabir, O. Chae, "Robust facial expression recognition based on local directional pattern," ETRI Journal, Vol. 32, No. 5, pp. 784-794, 2010.

[16] Z. Sun, G. Bebis, X. Yuan, S. J. Louis, "Genetic feature subset selection for gender classification: A comparison study," in Proceeding of the IEEE Workshop on Applications of Computer Vision, 2002, pp. 165-170.

[17] A. Jain, J. Huang, "Integrating independent components and linear discriminant analysis for gender classification," in Proceeding of the International Conference on Automatic Face and Gesture Recognition, 2004, pp. 159-163.

[18] M. Lyons, J. Budynek, A. Plante, S. Akamatsu, "Classifying facial attributes using a 2-d gabor wavelet representation and discriminant analysis," in Proceeding of the International Conference on Automatic Face and Gesture Recognition, 2000, pp. 202-207.

[19] S. Zhao, Y. Gao, B. Zhang, "Sobel-lbp," in Proceeding of the IEEE International Conference on Image Processing, 2008, pp. 2144-2147.

[20] T. Jabid, M. H. Kabir, O. Chae, "Local directional pattern for face recognition," in Proceeding of the IEEE International Conference on Consumer Electronics, 2010, pp. 329-330.

[21] F. Ahmed, M. H. Kabir, "Directional ternary pattern (dtp) for facial expression recognition," in Proceeding of the IEEE International Conference on Consumer Electronics, 2012, pp. 265-266.

[22] H. Kabir, T. Jabid, O. Chae, "Local directional pattern variance (ldpv): A robust feature descriptor for facial expression recognition," International Arab Journal of Information Technology, Vol. 9, pp. 382-391, 2012.

[23] N. Sun, W. Zheng, C. Sun, C. Zou, L. Zhao, "Gender classification based on boosting local binary pattern," in Proceeding of the International Symposium on Neural Networks, No. 2, 2006, pp. 194-201.

[24] H. Lian, B. Lu, "Multi-view gender classification using local binary patterns and support vector machines," in Proceeding of the International Symposium on Neural Networks, No. 2, 2006, pp. 202-209.

[25] G. Zhao, M. Pietikainen, "Boosted multi-resolution spatiotemporal descriptors for facial expression recognition," Pattern Recognition Letters, Vol. 30, No. 12 , pp. 1117-1127, 2009.

[26] T. Jabid, M. H. Kabir, O. Chae, "Gender classification using local directional pattern (ldp)," in Proceeding of the International Conference on Pattern Recognition, 2010 , pp. 2162-2165.

[27] F. Ahmed, E. Hossain, "Automated facial expression recognition using gradient-based ternary texture patterns," Chinese Journal of Engineering, Vol. 2013 Article ID 831747, 2013

[28] P. Phillips, H. Wechler, J. Huang, P. Rauss, "The fere database and evaluation procedure for face recognition algorithms," Image and Vision Computing, Vol. 16, No. 10, pp. 295-306, 1998.

[29] T. Ojala, M. Pietikainen, T. Maenpaa, "Multiresolution gray-scale and rotation invariant texture classification with local binary patterns," IEEE Transactions on Pattern Analysis and Machine Intelligence, Vol. 24, No. 7, pp. 971-987, 2002.

[30] T. Ahonen, A. Hadid, M. Pietikainen, "Face description with local binary patterns: Application to face recognition," IEEE Transaction on Pattern Analysis and Machine Intelligence, Vol. 28, No. 12, pp. 2037-2041, 2006 .

[31] C. Shan, S. Gong, P. W. McOwan, "Facial expression recognition based on local binary patterns: A comprehensive study," Image and Vision Computing, Vol. 27, No. 6, pp. 803-816, 2009.

[32] D. He, N. Cercone, "Local triplet pattern for contentbased image retrieval," in Proceeding of the International Conference on Image Analysis and Recognition, 2009, pp. 229-238.

[33] S. Gundimada, V. K. Asari, "Facial Recognition Using Multisensor Images Based on Localized Kernel Eigen Spaces," IEEE Transactions on Image Processing, Vol. 18, No. 6, pp. 1314-1325, 2009. 